\title{
A note on the Königs domain of compact composition operators on the Bloch space
}

\author{
Matthew M Jones
}

Correspondence: m.m.jones@mdx. ac.uk

Department of Mathematics, Middlesex University, The Burroughs, London, NW4 4BT, UK

\begin{abstract}
Let $\mathbb{D}$ be the unit disk in the complex plane. We define $\mathcal{B}_{0}$ to be the little Bloch space of functions $f$ analytic in $\mathbb{D}$ which satisfy $\lim _{|z| \rightarrow 1}\left(1-|z|^{2}\right)|f(z)|=0$. If $\varphi: \mathbb{D} \rightarrow \mathbb{D i s}$ analytic then the composition operator $C_{\phi}: f \mapsto f \circ \phi$ is a continuous operator that maps $\mathcal{B}_{0}$ into itself. In this paper, we show that the compactness of $C_{\phi}$ , as an operator on $\mathcal{B}_{0}$, can be modelled geometrically by its principal eigenfunction. In particular, under certain necessary conditions, we relate the compactness of $C_{\phi}$ to the geometry of $\Omega=\sigma(\mathbb{D})$, where $\sigma$ satisfies Schöder's functional equation $\sigma \circ \phi=$ $\phi^{\prime}(0) \sigma$.

2000 Mathematics Subject Classification: Primary 30D05; 47B33 Secondary 30D45.
\end{abstract}

\section{Introduction}

Let $\mathbb{D}=\{z \in \mathbb{C}:|z|<1\}$ be the unit disk in the complex plane and $\mathbb{T}$ its boundary. We define the Bloch space $\mathcal{B}$ to be the Banach space of functions, $f$, analytic in $\mathbb{D}$ with

$$
\|f\|_{\mathcal{B}}=|f(0)|+\sup _{z \in \mathbb{D}}\left(1-|z|^{2}\right)\left|f^{\prime}(z)\right|<\infty .
$$

This space has many important applications in complex function theory, see [1] for an overview of many of them. We denote by $\mathcal{B}_{0}$ the little Bloch space of functions in $\mathcal{B}$ that satisfy $\lim _{|z| \rightarrow 1}\left(1-|z|^{2}\right)\left|f^{\prime}(z)\right|=0$. This space coincides with the closure of the polynomials in $\mathcal{B}$.

Suppose now that $\varphi: \mathbb{D} \rightarrow \mathbb{D}$ is analytic, then we may define the operator, $C_{\phi}$, acting on $\mathcal{B}_{0}$ as $f \mapsto f \circ \phi$. It was shown in [2] that every such operator maps $\mathcal{B}_{0}$ continuously into itself. Moreover, it was proved that $C_{\phi}$ is compact on $\mathcal{B}_{0}$ if and only if $\phi$ satisfies

$$
\lim _{|z| \rightarrow 1} \frac{1-|z|^{2}}{1-|\varphi(z)|^{2}}\left|\varphi^{\prime}(z)\right|=0 .
$$

Recall that the hyperbolic geometry on $\mathbb{D}$ is defined by the distance

$$
\operatorname{disk}(z, w)=\inf \int_{\Gamma} \lambda_{\mathbb{D}}(\eta)|\mathrm{d} \eta|
$$

where the infimum is taken over all sufficiently smooth arcs that have endpoints $z$ and $w$.

\section{SpringerOpen ${ }^{\circ}$}

(C) 2011 Jones; licensee Springer. This is an Open Access article distributed under the terms of the Creative Commons Attribution License (http://creativecommons.org/licenses/by/2.0), which permits unrestricted use, distribution, and reproduction in any medium, provided the original work is properly cited. 
Here, $\lambda_{\mathbb{D}}(\eta)=\left(1-|\eta|^{2}\right)^{-1}$ is the Poincaré density of $\mathbb{D}$. The hyperbolic derivative of $\phi$ is given by $\phi^{\prime}(z) /\left(1-|\phi(z)|^{2}\right)$ and functions that satisfy (1) are called little hyperbolic Bloch functions or written $\varphi \in \mathcal{B}_{0}^{\mathcal{H}}$.

The Schröder functional equation is the equation

$$
\sigma \circ \varphi=\gamma \sigma .
$$

Note that this is just the eigenfunction equation for $C_{\phi}$. Konigs' theorem states that if $\phi$ has fixed point at the origin then (2) has a unique solution for $\gamma=\phi^{\prime}(0)$ which we call the Koenigs function and denote by $\sigma$ from here on. In the study of the geometric properties of $\phi$ in relation to the operator theoretic properties of $C_{\phi}$, it has become evident that the Kœnigs function is much more fruitful to study than $\phi$ itself. In particular, see [3] for a discussion of the Kœnigs function in relation to compact composition operators on the Hardy spaces.

If we let $\Omega=\sigma(\mathbb{D})$ be the Koenigs domain of $\phi$, then (2) may be interpreted as implying that the action of $\phi$ on $\mathbb{D}$ is equivalent to multiplication by $\gamma$ on $\Omega$. It is due to this that the pair $(\Omega, \gamma)$ is often called the geometric model for $\phi$.

In this paper, we study the geometry of $\Omega$ when $\varphi \in \mathcal{B}_{0}^{\mathcal{H}}$. In order to do this, we will use the hyperbolic geometry of $\Omega$. If $f: \mathbb{D} \rightarrow \Omega$ is a universal covering map and $\Omega$ is a hyperbolic domain in $\mathbb{C}$, then the Poincaré density on $\Omega$ is derived from the equation

$$
\lambda_{\Omega}(f(z))\left|f^{\prime}(z)\right|=\lambda_{\mathbb{D}}(z),
$$

which is independent of the choice of $f$. Since this equation, in terms of differentials, is $\lambda_{\Omega}(w)|d w|=\lambda_{\mathbb{D}}(z)|d z|$ (for $w=f(z)$ ), we see that the hyperbolic distance on $\mathbb{D}$ defined above carries over to a hyperbolic distance on $\Omega$. For a more thorough treatment of the hyperbolic metric, see [4].

In [5], the Königs domain of a compact composition operator on the Hardy space was studied and the following result was proved.

Theorem A. Let $\phi$ be a univalent self-map of $\mathbb{D}$ with a fixed point in $\mathbb{D}$. Suppose that for some positive integer $n_{0}$ there are at most finitely many points of $\mathbb{T}$ at which $\varphi_{n_{0}}$ has an angular derivative. Then the following are equivalent.

1. Some power of $C_{\phi}$ is compact on the Hardy space $H^{2}$;

2. $\sigma$ lies in $H^{p}$ for every $p<\infty$;

3. $\Omega=\sigma(\mathbb{D})$ does not contain a twisted sector.

Here, $\Omega$ is said to contain a twisted sector if there is an unbounded curve $\Gamma \in \Omega$ with

$$
\delta_{\Omega}(w) \geq \varepsilon|w|
$$

for some $\varepsilon>0$ and all $w \in \Gamma$, where $\delta_{\Omega}$ is the distance from $w$ to the boundary of $\Omega$ as defined below. The purpose of this paper is to provide a similar result to this in the context of the Bloch space.

\section{Simply connected domains}

Throughout this section, we assume that $\Omega$ is an unbounded simply connected domain in $\mathbb{C}$ with $0 \in \Omega$. As in the previous section, $\sigma$ represents the Riemann mapping of $\mathbb{D}$ 
onto $\Omega$ with $\sigma(0)=0$ and $\sigma^{\prime}(0)>0$. We will also define $\phi$ via the Schröder functional equation. Throughout we let

$$
\delta_{\Omega}(w)=\inf _{\zeta \notin \Omega}|w-\zeta|,
$$

so that $\delta_{\Omega}(w)$ is the Euclidean distance from $w$ to the boundary of $\Omega$.

Theorem 1. Let $\phi$ be a univalent function mapping $\mathbb{D} i n t o \mathbb{D}, \phi(0)=0$. Suppose that the closure of $\varphi(\mathbb{D})$ intersects $\mathbb{T}$ only at finitely many fixed points and is contained in a Stolz angle of opening no greater than $\alpha \pi$ there.

If $\left|\phi^{\prime}(0)\right|>16 \tan (\alpha \pi / 2)$ then the following are equivalent

$$
\begin{aligned}
& \text { 1. } C_{\phi} \text { is compact on } \mathcal{B} \text {; } \\
& \text { 2. } \lim _{\substack{w \rightarrow \infty \\
w \in \gamma}} \frac{\delta_{\Omega}(w)}{\delta_{\Omega}(\gamma w)}=0 ; \\
& \text { 3. For every } n>0, \sigma^{n} \in \mathcal{B}_{0} \text {. }
\end{aligned}
$$

Remark: It has recently been shown by Smith [6] that compactness of $C_{\phi}$ on $\mathcal{B}$ is equivalent to compactness of $C_{\phi}$ on $\mathcal{B}_{0}, B M O A$ and $V M O A$ when $\phi$ is univalent and so in the above theorem, the first condition could read: $C_{\phi}$ is compact on $\mathcal{B}, \mathcal{B}_{0}, B M O A$ and $V M O A$ Before proceeding, we prove the following lemma.

Lemma 1. Under the hypotheses of the theorem, $w$ and $\gamma w$ tend to the same prime end at $\infty$, and $\partial \gamma \Omega \subset \Omega$.

Proof. The first assertion follows from the fact that the closure of $\varphi(\mathbb{D})$ touches $\mathbb{T}$ only at fixed points. Suppose now that the second assertion is false and there are distinct prime ends $\rho_{1}$ and $\rho_{2}$ with $\rho_{1}=\gamma \rho_{2}$. Then under the boundary correspondence given by $\sigma$ there are distinct points $\eta, \zeta \in \mathbb{T}$ with

$$
\sigma(\eta)=\gamma \sigma(\zeta)=\sigma(\varphi(\zeta))
$$

It follows that $\varphi(\zeta) \in \mathbb{T}$ and therefore $\zeta$ is a fixed point of $\phi$. Hence, we have the contradiction $\rho_{1}=\rho_{2}$.

Proof. We first prove that 1 is equivalent to 2 .

By the results of Madigan and Matheson [2], and Smith [6] cited above $C_{\phi}$ is compact on $\mathcal{B}$ if and only if

$$
\lim _{|z| \rightarrow 1} \frac{1-|z|^{2}}{1-|\varphi(z)|^{2}}\left|\varphi^{\prime}(z)\right|=0 .
$$

However, by Schröder's equation

$$
\begin{aligned}
\frac{1-|z|^{2}}{1-|\varphi(z)|^{2}}\left|\varphi^{\prime}(z)\right| & =\frac{\lambda_{\mathbb{D}}(\varphi(z))}{\lambda_{\mathbb{D}}(z)}\left|\varphi^{\prime}(z)\right| \\
& =\frac{\lambda_{\Omega}(\sigma \circ \varphi(z))}{\lambda_{\Omega}(\sigma(z))} \frac{\left|\sigma^{\prime} \circ \varphi(z) \varphi^{\prime}(z)\right|}{\left|\sigma^{\prime}(z)\right|} \\
& =|\gamma| \frac{\lambda_{\Omega}(\gamma w)}{\lambda_{\Omega}(w)}
\end{aligned}
$$

Since $\Omega$ is simply connected, $\lambda_{\Omega}(w) \otimes 1 / \delta_{\Omega}(w)$ and so $C_{\phi}$ is compact on $\mathcal{B}$ if and only if 


$$
\lim _{w \rightarrow \partial \Omega} \frac{\delta_{\Omega}(w)}{\delta_{\Omega}(\gamma w)}=0 .
$$

Since $\gamma \Omega \subset \Omega, \gamma w \rightarrow \partial \Omega$ implies that $w \rightarrow \partial \Omega$. Therefore, (3) holds if and only if

$$
\lim _{\gamma w \rightarrow \partial \Omega} \frac{\delta_{\Omega}(w)}{\delta_{\Omega}(\gamma w)}=0 .
$$

By the Lemma, we see that $\gamma w \rightarrow \partial \Omega$ means $w \rightarrow \infty$ and $w \in \gamma \Omega$, and we have shown that 1 and 2 are equivalent.

Suppose that 2 holds and let $\varepsilon>0$ be given. Then we can find a $R>0$ so that $\delta_{\Omega}(w)$ $<\varepsilon \delta_{\Omega}(\gamma w)$ for all $|w|>R$, since there are only a finite number of prime ends at $\infty$. Choose $w \in \Omega$ arbitrarily with modulus greater than $R$ and let $n$ satisfy $|\gamma|^{-n} R<|w| \leq$ $|\gamma|^{-n-1} R$.

Then we have that $\delta_{\Omega}(w)<\varepsilon^{n} \delta_{\Omega}\left(\gamma^{n} w\right)$ and hence

$$
\frac{-\log \delta_{\Omega}(w)}{\log |w|}>\frac{-n \log \varepsilon-\log \delta_{\Omega}\left(\gamma^{n} w\right)}{-(n+1) \log |\gamma|+\log R} .
$$

Now as $w \rightarrow \infty$ in $\gamma \Omega, \gamma^{n} w$ lies in a closed set properly contained in $\Omega$ and therefore $\delta_{\Omega}\left(\gamma^{n} w\right)$ is bounded below by a constant independent of $w$. We thus have that

$$
\liminf _{w \rightarrow \infty} \frac{-\log \delta_{\Omega}(w)}{\log |w|}>\frac{-\log \varepsilon}{-\log |\gamma|}
$$

and since $\varepsilon$ was arbitrary, the left-hand side of the above inequality must tend to $\infty$. Hence, we have shown that $\lim _{w \rightarrow \infty}|w|^{\beta} \delta_{\Omega}(w)=0$ for every $\beta>0$.

Now $\sigma^{n} \in \mathcal{B}_{0}$ may be interpreted geometrically as $\lim _{w \rightarrow \partial \Omega} n|w|^{n-1} \delta_{\Omega}(w)=0$ and this follows from the above argument. Therefore, 2 implies 3.

To show that 3 implies 2, we need to show that if

$$
\lim _{w \rightarrow \infty} f(w)=\lim _{w \rightarrow \infty} \frac{-\log \delta_{\Omega}(w)}{\log |w|}=\infty
$$

then 2 holds.

To complete the proof, we require the following lemma whose proof we merely sketch.

Lemma 2. Under the hypotheses of the theorem,

$$
\limsup _{w \rightarrow \infty} \frac{\delta_{\Omega}(w)}{\delta_{\Omega}(\gamma w)} \leq K<1 .
$$

Sketch of Proof. First note that

$$
\limsup _{|w| \rightarrow 1} \frac{\delta_{\Omega}(w)}{\delta_{\Omega}(\gamma w)} \leq \frac{16}{\left|\varphi^{\prime}(0)\right|} \limsup _{|z| \rightarrow 1} \frac{\delta_{\varphi(\mathbb{D})}(z)}{\delta_{\mathbb{D}}(z)} .
$$

Now if $\varphi(\mathbb{D})$ lies in a non-tangential angle of opening $\alpha \pi$ at $\zeta$, then a short calculation shows that

$$
\limsup _{z \rightarrow \zeta} \frac{\delta_{\varphi(\mathbb{D})}(z)}{\delta_{\mathbb{D}}(z)} \leq \tan \frac{\alpha \pi}{2}
$$

and the assertion follows. 
Now with $f$ defined above, we have

$$
\begin{aligned}
f(\gamma w)-f(w) & =\frac{-\log \delta_{\Omega}(\gamma w)}{\log |\gamma w|}-\frac{-\log \delta_{\Omega}(w)}{\log |w|} \\
& \sim \frac{\log \delta_{\Omega}(w) / \delta_{\Omega}(\gamma w)}{\log |w|}<0
\end{aligned}
$$

for large enough $w$. Hence,

$$
\frac{\delta_{\Omega}(w)}{\delta_{\Omega}(\gamma w)}=\frac{|\gamma|^{f(\gamma w)}}{|w|^{f(w)-f(\gamma w)}} \leq|\gamma|^{f(\gamma w)} \rightarrow 0
$$

as $w \rightarrow \infty$ and so 2 holds.

It is of interest to consider the growth of $\sigma$ since condition 3 would imply that it has very slow growth. The following corollary follows from 3 and the fact that functions in $\mathcal{B}_{0}$ grow at most of order $\log 1 /(1-|z|)$.

Corollary 1. Suppose that $\phi$ satisfies the hypotheses of the Theorem and that any of the equivalent conditions holds, then for $r=|z|$.

$$
\log |\sigma(z)|=o\left(\log \log \frac{1}{1-r}\right) .
$$

We also provide the following restatement of the hypotheses of Theorem 1 to illustrate the main properties of the Königs domain.

Corollary 2. Let $\Omega$ be an unbounded domain in $\mathbb{C}$ with $\gamma \Omega \subset \Omega$ and $0 \in \Omega$. Suppose that has $\Omega$ only finitely many prime ends at $\infty$ and

$$
\limsup _{w \rightarrow \infty} \frac{\delta_{\Omega}(w)}{\delta_{\Omega}(\gamma w)}<1 .
$$

In addition, suppose that $\partial \gamma \Omega \subset \Omega$. If $\sigma: \mathbb{D} \rightarrow \Omega, \sigma(0)=0, \sigma^{\prime}(0)>0$, and $\phi$ is defined by Schröder's equation, then the following are equivalent.

$$
\begin{aligned}
& \text { 1. } C_{\phi} \text { is compact on } \mathcal{B} \text {; } \\
& \text { 2. } \lim _{\substack{w \rightarrow \infty \\
w \in \gamma}} \frac{\delta_{\Omega}(w)}{\delta_{\Omega}(\gamma w)}=0 \text {; } \\
& \text { 3. For every } n>0, \sigma^{n} \in \mathcal{B}_{0} \text {. }
\end{aligned}
$$

The hypothesis on the boundary of $\Omega$ is vital. If we do not assume that $\partial \gamma \Omega \subset \Omega$, then we deduce from the proof of the Theorem that $\varphi \in \mathcal{B}_{0}^{\mathcal{H}}$ is equivalent to

$$
\lim _{\gamma w \rightarrow \partial \Omega} \frac{\delta_{\Omega}(w)}{\delta_{\Omega}(\gamma w)}=0 .
$$

In this situation, the finite part of the boundary of $\Omega$ plays a complicated role in the behaviour of $\phi$. We conclude this section by constructing a domain that displays very bad boundary properties. This answers a question of Madigan and Matheson in [2].

In [2] it was shown that if $\partial \phi(D)$ touches $\mathbb{T}=\partial \mathbb{D}$ in a cusp, then $\varphi \in \mathcal{B}_{0}^{\mathcal{H}}$. However, it is not sufficient that $\partial \phi(D)$ touches $\mathbb{T}$ at an angle greater that 0 . The question was raised of whether or not it is possible that $\overline{\varphi(D)} \cap \mathbb{\mathbb { V }}$ can be infinite. 
With the hypothesis that $\partial \gamma \Omega \subset \Omega$ the prime ends at $\infty$ correspond to points of $\overline{\varphi(D)}$ that touch $\mathbb{T}$. Therefore, $\overline{\varphi(D)} \cap \mathbb{T}$ is at most countable. A natural question to ask is whether or not $\Lambda \overline{(\varphi(D)} \cap \mathbb{T})$ can ever be positive, where $\Lambda$ represents linear measure.

This example is well known in the setting of the unit disk, see [7, Corollary 5.3]. We describe here the construction in terms of the Königs domain.

Theorem 2. There is a univalent function $\varphi \in \mathcal{B}_{0}^{\mathcal{H}}$ such that $\overline{\varphi(D)} \cap \mathbb{T}=\mathbb{T}$.

Proof. We construct the domain $\Omega$ so that it satisfies (4). Let $0<\gamma<1$ be given. We will define a nested sequence $\Theta_{n} \subset \mathbb{T}, n=1,2, \ldots$ so that

$$
\partial \Omega=\cup_{n \geq 1}\left\{r e^{i \theta}: \gamma^{-n} \leq r<\infty, \theta \in \Theta_{n}\right\},
$$

where $\Theta_{n} \subset \Theta_{n+1}$ for all $n=1,2, \ldots$

First let $N>2$ be chosen arbitrarily and let $\Theta_{1}=\{2 \pi k / N: k=0, \ldots, N-1\}$.

Suppose now that $\Theta_{n}$ has been defined, then let $\Theta_{n+1}$ be such that $\Theta_{n} \subset \Theta_{n+1}$ and whenever $\theta \in \Theta_{n}$ is isolated, we define a sequence $\theta_{k} \in \Theta_{n+1}, k=1,2, \ldots$, so that $\theta_{k} \rightarrow$ $\theta$ as $k \rightarrow \infty$ and for each $k$ there is a $j$ so that $\theta-\theta_{k}=\theta_{j-\theta}$. Moreover, assume that

$$
\lim _{k \rightarrow \infty} \frac{\theta_{k+1}-\theta_{k}}{\left(\theta-\theta_{k}\right)^{2}}=0
$$

In this way, we define the sequence of sets $\Theta_{n}, n=1,2, \ldots$ We will, furthermore, assume that for each $e^{i \theta} \in \mathbb{T}$, there is a sequence $\theta_{n} \in \Theta_{n}, n=1,2, \ldots$, such that $\theta_{n} \rightarrow$ $\theta$.

We claim that this gives the desired domain $\Omega$ with boundary defined by (5).

To see this, let $\gamma w \in \Omega$ be arbitrary, then by construction, we may find a $\zeta \in \partial \Omega$ so that $\delta_{\Omega}(\gamma w)=|\zeta-\gamma w|$. It is readily seen that for such $\zeta$, there is an $n$ so that $\zeta \in\left\{r e^{\mathrm{i} \theta}\right.$ : $\left.r \geq \gamma^{-n}\right\}$ for some $\theta \in \Theta_{n}$ and moreover, $\theta$ is isolated in $\Theta_{n}$.

If we now consider $w$, we may find a sequence $\theta_{k} \rightarrow \theta$ as $k \rightarrow \infty$ so that $\left\{r e^{i \theta_{k}}: r \geq \gamma^{-n-1}\right\} \in \partial \Omega$ for all $k$ hence we may fix a $k$ so that $\delta_{\Omega}(w)=|w-n|$ for $\eta=r e^{i \theta_{k}}$.

By estimating the line segment $[w, \eta]$ by the arc of $r \mathbb{1}$ joining $w$ to $\eta$, we see that $\delta_{\Omega}$ $(w) \bigotimes|w|\left|\alpha-\theta_{k}\right|$ where $w=r e^{\mathrm{i} \alpha}$.Therefore, we have the estimate $\delta_{\Omega}(w) \leq|w| \mid \theta_{k+1}$ $\theta_{k} \mid$. By a similar argument, we deduce the estimate $\delta_{\Omega}(\gamma w) \otimes|\gamma w|\left|\theta-\theta_{k}\right|$ and so

$$
\frac{\delta_{\Omega}(w)}{\delta_{\Omega}(\gamma w)} \leq \gamma^{-1}\left|\frac{\theta_{k+1}-\theta_{k}}{\theta-\theta_{k}}\right| \leq \gamma^{-1}\left|\theta-\theta_{k}\right|
$$

by (6) and so the construction is complete.

We claim that if $\sigma: \mathbb{D} \rightarrow \Omega$ is defined as usual and $\phi$ is given by Schröder's equation, then $\overline{\varphi(\mathbb{D})} \cap \mathbb{T}=\mathbb{T}$.

In fact, if $\theta \in \Theta_{n}$ is isolated, then the ray $R=\left\{r e^{\mathrm{i} \theta}: r \geq \gamma^{-n-1}\right\}$ is contained in a single prime end of $\Omega$. Therefore, to each such ray, there exists a point $\zeta \in \mathbb{T}$ that corresponds to $R$ under $\sigma$. Since $\gamma R \subset \partial \Omega$, we thus have that $\zeta$ corresponds to a prime end $p$ under $\phi$ with $p \cap \mathbb{\mathbb { V }} \neq \emptyset$.

On the other hand, if $\theta \in \Theta_{n}$ is isolated, then $R^{\prime}=\left\{r e^{\mathrm{i} \theta}: \gamma^{-n} \leq r<\gamma^{-n-1}\right\}$ satisfies $\gamma R^{\prime} n$ $\partial \Omega=\varnothing$, and so there is an $\operatorname{arc} \rho_{\theta} \subset \mathbb{D}$ such that $\sigma\left(\rho_{\theta}\right)=R^{\prime}$ and $\rho_{\theta}$ has an end-point in $\mathbb{T}$. 
Hence, each $\eta \in \mathbb{T}$ is contained in a prime end of $\varphi(\mathbb{D})$ and

$$
\varphi(\mathbb{D})=\mathbb{D} \backslash \bigcup_{\theta \in \Theta_{n} \text { isolated }} \rho_{\theta} .
$$

The result follows.

\section{Multiply connected domains}

The geometric arguments of the previous section potentially lend themselves to multiply connected domains in the following way. Suppose that $\Omega$ is a domain in $\mathbb{C}$ with 0 $\in \Omega$ and $\gamma \Omega \subset \Omega$ for some $\gamma \in \mathbb{D} \backslash\{0\}$. Let $\sigma$ be a universal covering map of $\mathbb{D}$ onto $\Omega$ with $\sigma(0)=0$. Then $\sigma^{\prime}(0) \neq 0$ and we may define $\phi$ via (2). Now we have

$$
\frac{1-|z|^{2}}{1-|\varphi(z)|^{2}}\left|\varphi^{\prime}(z)\right|=|\gamma| \frac{\lambda_{\Omega}(\gamma w)}{\lambda_{\Omega}(w)} .
$$

However, if $\Omega$ is not simply connected, then $\sigma$ is an infinitely sheeted covering of $\Omega$ and therefore the equation $\sigma(z)=0$ has infinitely many distinct solutions, $z_{n}, n=0,1$, …

Now, since

$$
\frac{1-\left|z_{n}\right|^{2}}{1-\left|\varphi\left(z_{n}\right)\right|^{2}}\left|\varphi^{\prime}\left(z_{n}\right)\right|=|\gamma|>0
$$

for all $n \geq 0$, we see that $\varphi \notin \mathcal{B}_{0}^{\mathcal{H}}$. Thus, we have proved the following result.

Proposition 1. Suppose that $\Omega \subset \mathbb{C}$ is a domain satisfying $0 \in \Omega$ and $\gamma \Omega \subset \Omega$, and let $\sigma: \mathbb{D} \rightarrow$ Sbe a universal covering map with $\sigma(0)=0$.

If $\phi$, as defined by (2) is in $\mathcal{B}_{0}^{\mathcal{H}}$ then $\Omega$ is simply connected.

\section{Competing interests}

The author declares that they have no competing interests.

Received: 31 January 2011 Accepted: 10 August 2011 Published: 10 August 2011

\section{References}

1. Anderson, JM: Bloch Functions: The Basic Theory. Operators and Function Theory. D Reidel. 1-17 (1985)

2. Madigan, K, Matheson, A: Compact Composition Operators on the Bloch Space. Trans Am Math Soc. 347, 2679-2687 (1995). doi:10.2307/2154848

3. Shapiro, JH: Composition Operators and Classical Function Theory. Springer. (1993)

4. Ahlfors, LV: Conformal Invariants, Topics in Geometric Function Theory. McGraw-Hill. (1973)

5. JH, Shapiro, W, Smith, A, Stegenga: Geometric models and compactness of composition operators. J Funct Anal. 127 21-62 (1995). doi:10.1006/jfan.1995.1002

6. Smith, W: Compactness of composition operators on BMOA. Proc Am Math Soc. 127, 2715-2725 (1999). doi:10.1090/ S0002-9939-99-04856-X

7. Bourdon, P, Cima, J, Matheson, A: Compact composition operators on BMOA. Trans Am Math Soc. 351, 2183-2196 (1999). doi:10.1090/S0002-9947-99-02387-9 